\title{
Use of segmented CT transmission map to avoid metal artifacts in PET images by a PET-CT device
} Siroos Mirzaei*1,2, Michel Guerchaft ${ }^{3}$, Christopher Bonnier ${ }^{1}$, Peter Knoll ${ }^{2}$, Michel Doat ${ }^{1}$ and Peter Braeutigam ${ }^{1}$

\author{
Address: ${ }^{1}$ Centre National PET, Clinique Ste. Thérèse, Luxembourg, ${ }^{2}$ Wilhelminenspital, Institute of Nuclear Medicine, Vienna, Austria and \\ ${ }^{3}$ Philips Medical Systems, BeNeLux \\ Email: Siroos Mirzaei* - mirzaei@gmx.at; Michel Guerchaft - michel.guerchaft@philips.com; Christopher Bonnier - bonnierchristo@yahoo.fr; \\ Peter Knoll - peter.knoll@wienkav.at; Michel Doat - doatjetm@pt.lu; Peter Braeutigam - pbab@gmx.de \\ * Corresponding author
}

Published: 14 June 2005

BMC Nuclear Medicine 2005, 5:3 doi:10.1186/147/-2385-5-3
Received: 25 October 2004

Accepted: 14 June 2005

This article is available from: http://www.biomedcentral.com/l47/-2385/5/3

(c) 2005 Mirzaei et al; licensee BioMed Central Ltd.

This is an Open Access article distributed under the terms of the Creative Commons Attribution License (http://creativecommons.org/licenses/by/2.0), which permits unrestricted use, distribution, and reproduction in any medium, provided the original work is properly cited.

\begin{abstract}
Background: Attenuation correction is generally used to PET images to achieve count rate values independent from tissue densities. The goal of this study was to provide a qualitative comparison of attenuation corrected PET images produced by a PET-CT device (CT, $120 \mathrm{kV}, 40 \mathrm{mAs}$, FOV 600 $\mathrm{mm})$ with and without segmentation of transmission data (ACseg ${ }^{+}$and $\mathrm{ACseg-respectively).}$ Methods: The reconstructed images were compared to attenuation corrected images obtained with a high-energy transmission source (Cs-137-662 keV).

Thirty oncologic patients were studied using $C T$ and ${ }^{137} \mathrm{Cs}$ for attenuation correction. All image data were acquired using the Gemini PET-CT scanner (Philips Medical Systems). It is an open PETCT system that consists of the MX8000 multislice CT and the Allegro PET scanner arranged in a separable configuration. Images with $\mathrm{ACseg}^{+}$and $\mathrm{ACseg}$ were analyzed simultaneously in coronal, sagittal and transaxial planes. Two nuclear medicine physicians reviewed the image sets. Results: The image quality in the area of metal implants was better with $\mathrm{ACseg}^{+}$than $\mathrm{ACseg}$, without metal induced artifacts generally observed in CT corrected images. Further the images with $\mathrm{ACseg}^{+}$were qualitatively comparable to those obtained with ${ }^{137} \mathrm{Cs}$ attenuation correction. Conclusions: In case of metal implants, PET studies corrected by CT should preferably use the $\mathrm{ACseg}^{+}$method to avoid the image artifacts.
\end{abstract}

\section{Background}

Attenuation correction (AC) is generally applied to achieve count rate values independent from tissue densities. Transmission scanning was already suggested in 1952 by Myneord [1]. The transmission scanning was used to match anatomical contours to the radionuclide distribution measured by a rectilinear scanner [2]. Different isotopes have been used to acquire transmission data as earlier I-125, I-131, Tm-170 and later Tc-99 m, I-131, Ba-
133, Cs-137 and Ge-68. With the development of SPECT and PET systems the transmission scanning was used for the purpose of $\mathrm{AC}$ and various methods for the use of transmission data were developed $[3,4]$. The PET transmission scan is ideally acquired before the radiopharmaceutical is administered, but would lead to long clinical protocols which are not suitable for routine use. Today, for PET-investigations post-injection transmission scans are performed using electronically windowed rotating rod 


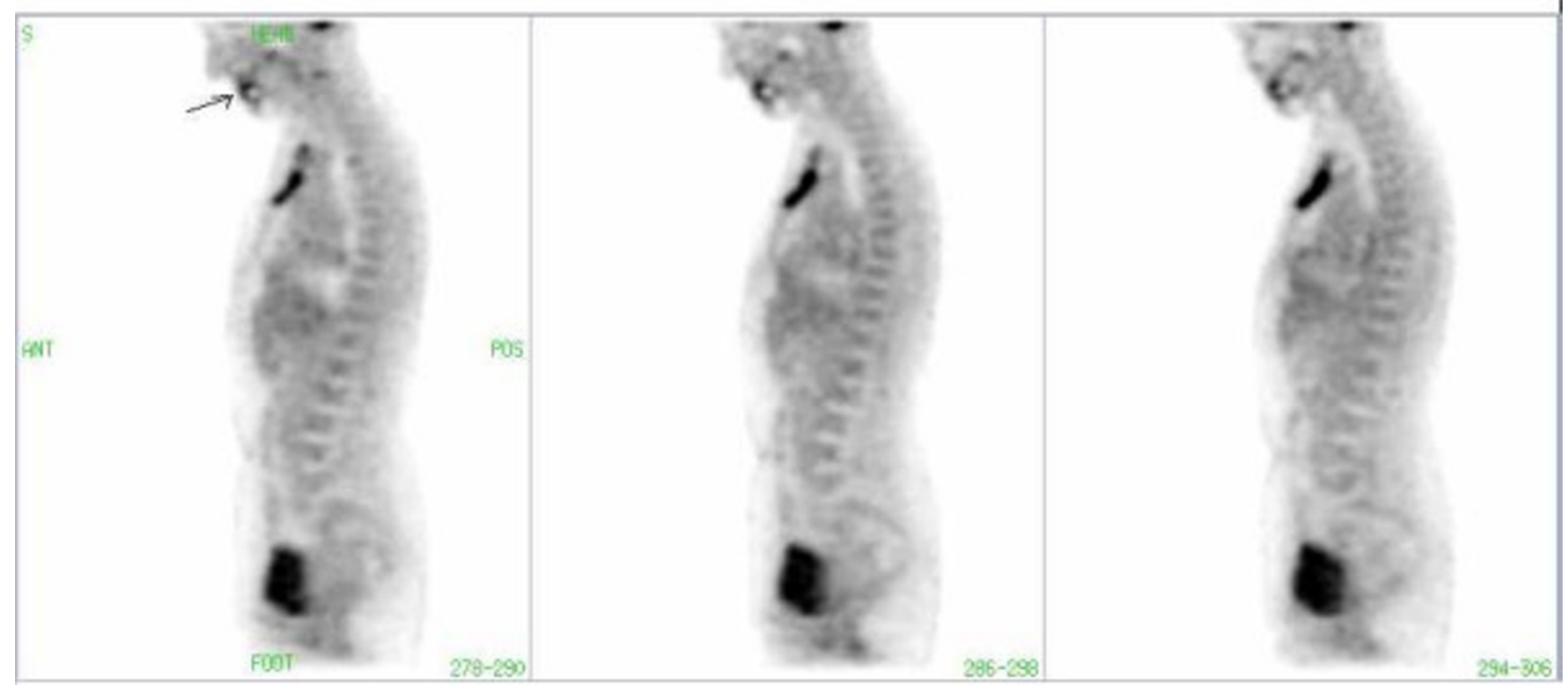

Figure I

FDG-PET image of a patient with a hypermetabolic retrosternal mass. Artifact (arrow) due to a dental implant in CT corrected images without segmentation.

sources as Ge-68, or rotating point sources as Cs-137 or CT in PET-CT systems. The combined PET-CT systems have the advantage to provide anatomical information while CT transmission data can be used for $\mathrm{AC}$ of the emission scan. This results in getting structural and metabolic information in the same session and a joint report of a nuclear medicine physician and a radiologist is then sent to the referring physician. However the PET examinations corrected for attenuation by CT images may be hampered by artifacts which are not usually seen in only PET images, as respiration [5], truncation [6], CT contrast agents and metal implants [7-9]. It is known that the high atomic numbers of contrast agents or metal implants result in increased fraction of photelectric interactions, yielding increased Housfield units (HU). With the current used reconstruction algorithms, contrast can be therefore missclassified as high density bone and thus associated with an incorrect scaling factor [10]. The goal of this study was to provide a qualitative comparison of attenuation corrected PET images of patients with metal implants produced by a PET-CT device with and without segmentation of CT.

\section{Methods}

Thirty oncologic patients with metal implants (hip prosthesis, dental implants, pacemaker) were studied using CT and Cs-137 transmission scans for attenuation correction. None of the included patients had clinical signs of local inflammation of the implants. All image data were acquired using the Gemini Dual PET-CT scanner (Philips Medical systems). The GEMINI Dual is an open PET-CT system that combines a helical dual slice CT and a 3D PET scanner equipped with its own transmission source [11]. Gantries are arranged in a separable configuration that allows the use of the 2 scanners separately when desired. The MX8000 EXP CT scanner is a dual slice system whose detector consist of 1344 Cadmium Tungstate elements. Gantry allows a patient port of $70 \mathrm{~cm}$. Minimum scan time per full rotation is $0.5 \mathrm{~s}$. and slice thickness can range between $0.5 \mathrm{~mm}$ and $10 \mathrm{~mm}$. The Allegro 3D PET scanner works exclusively in $3 \mathrm{D}$ detection mode (no septa). It is comprised of 29 arrays of 616 GSO (gadolinium oxyorthosilicate) crystals each. Crystal dimensions are $4 \times 6 \times$ $20 \mathrm{~mm}^{3}$. The axial field of view is $180 \mathrm{~mm}$ and patient port is $63 \mathrm{~cm}$. The rotating point source of Cs-137 located in the gantry allows acquisition of transmission scans in 


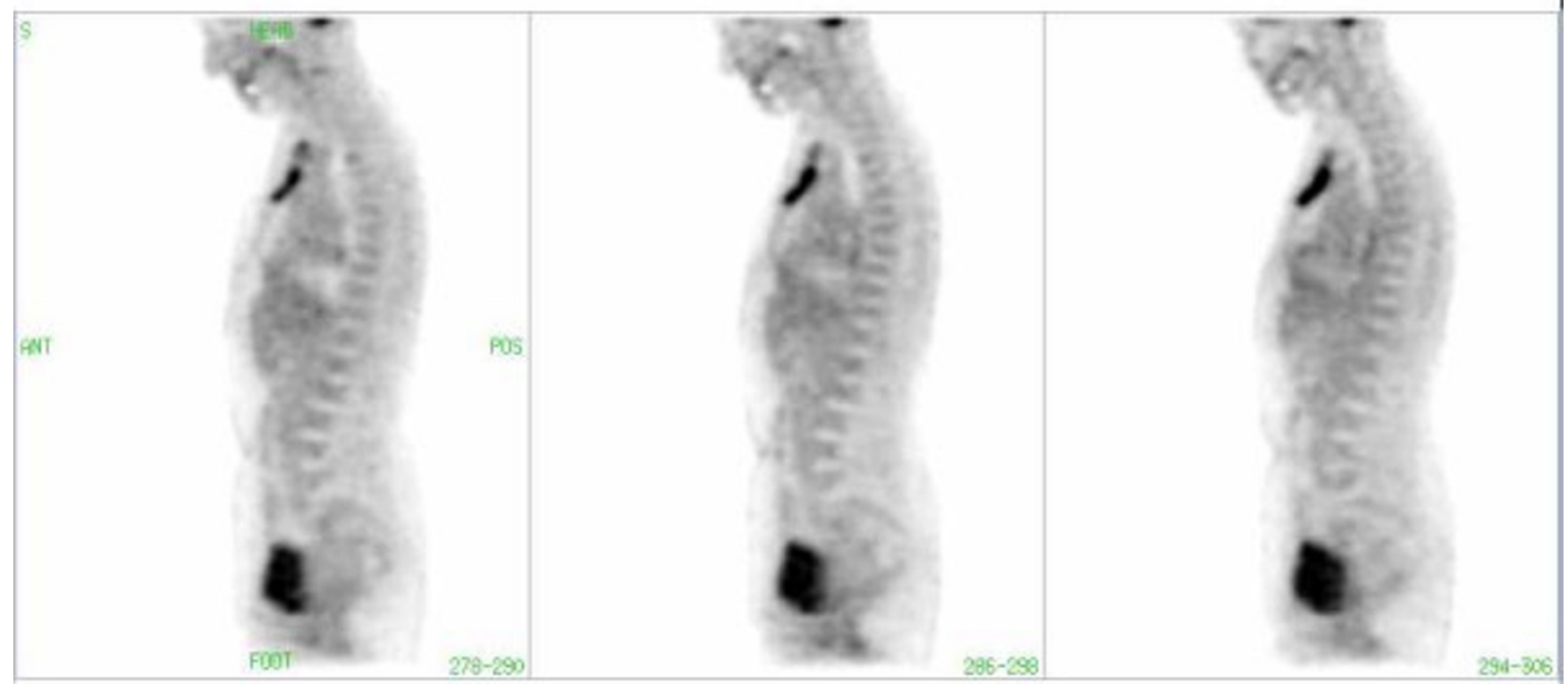

Figure 2

The same patient as in Fig. I: The Artifact due to dental mass (Fig. I) is not visible in the CT-corrected images with segmentation of transmission data.

3D detection mode. Cs-137 emits single photons with energy of $662 \mathrm{KeV}$.

Patients were scanned 1 hour after injection of $4 \mathrm{MBq} / \mathrm{Kg}$ FDG. A low dose CT helical scan was performed first (scan field of $600 \mathrm{~mm}$, increment of $5 \mathrm{~mm}$, slice thickness 6.5 $\mathrm{mm}$, pitch of $1.5,0.75$ second per rotation, matrix $512 \times$ $512,120 \mathrm{KV}, 40 \mathrm{mAs}$ ), followed by the Cs-137 transmission scan. The high activity of the Cs-137 source (750 $\mathrm{MBq}$ - half-life 30 years) and the detection of single events allows fast transmissions scan. Total duration of Cs-137 transmission scan is about 4 minutes $(100 \mathrm{~cm}$ scan length). PET emission scan is automatically started at the end point of transmission. Emission scan consists of 8 to 11 bed positions of 3 minutes each which allows to cover $77 \mathrm{~cm}$ to $102 \mathrm{~cm}$. Randoms are online subtracted during acquisition (time-delayed events). Total acquisition time per patient varied from 30 to 40 minutes.

4 sets of data were reconstructed for each patient: no AC, AC with Cs-137 point source, AC with non-segmented CT image (Acseg) and AC with segmented CT (Acseg+). Acquisition data were processed with standard package delivered with the system (Petview software - Philips
Medical Systems). Reconstruction without AC was performed with RAMLA 2D iterative algorithm [12,13]. All reconstructions with AC were performed with RAMLA 3D iterative algorithm $[12,14]$. Voxel size after reconstruction is $4 \times 4 \times 4 \mathrm{~mm}^{3}$. Scatter correction is performed by fitting the tails of activity outside patient with a parabolic curve. AC with Cs-137 source is performed by segmentation of original transmission data set. Segmentation is used to divide the CT image into regions representing different tissue types (lung, soft tissue, bone). All pixels in regions corresponding to different tissues are assigned the value associated with these particular tissue types. Regions with higher attenuation coefficients than bone tissues (metal implants) are assigned to soft tissue attenuation coefficient. Natural variations in densities are reflected by merging $[15,16]$ segmented image with the measured attenuation map that is first corrected such that the 'average' values of lung tissue's and soft tissue's attenuation coefficients are equal to nominal values set in reconstruction protocol file. Merging is controlled by lung and soft tissues attenuation coefficients (user defined uniformity parameters). With values set to 1.0 , only the segmented image is used and no merging is performed. With values set to 0.0 , only an over-smoothed mean-adjusted image of 


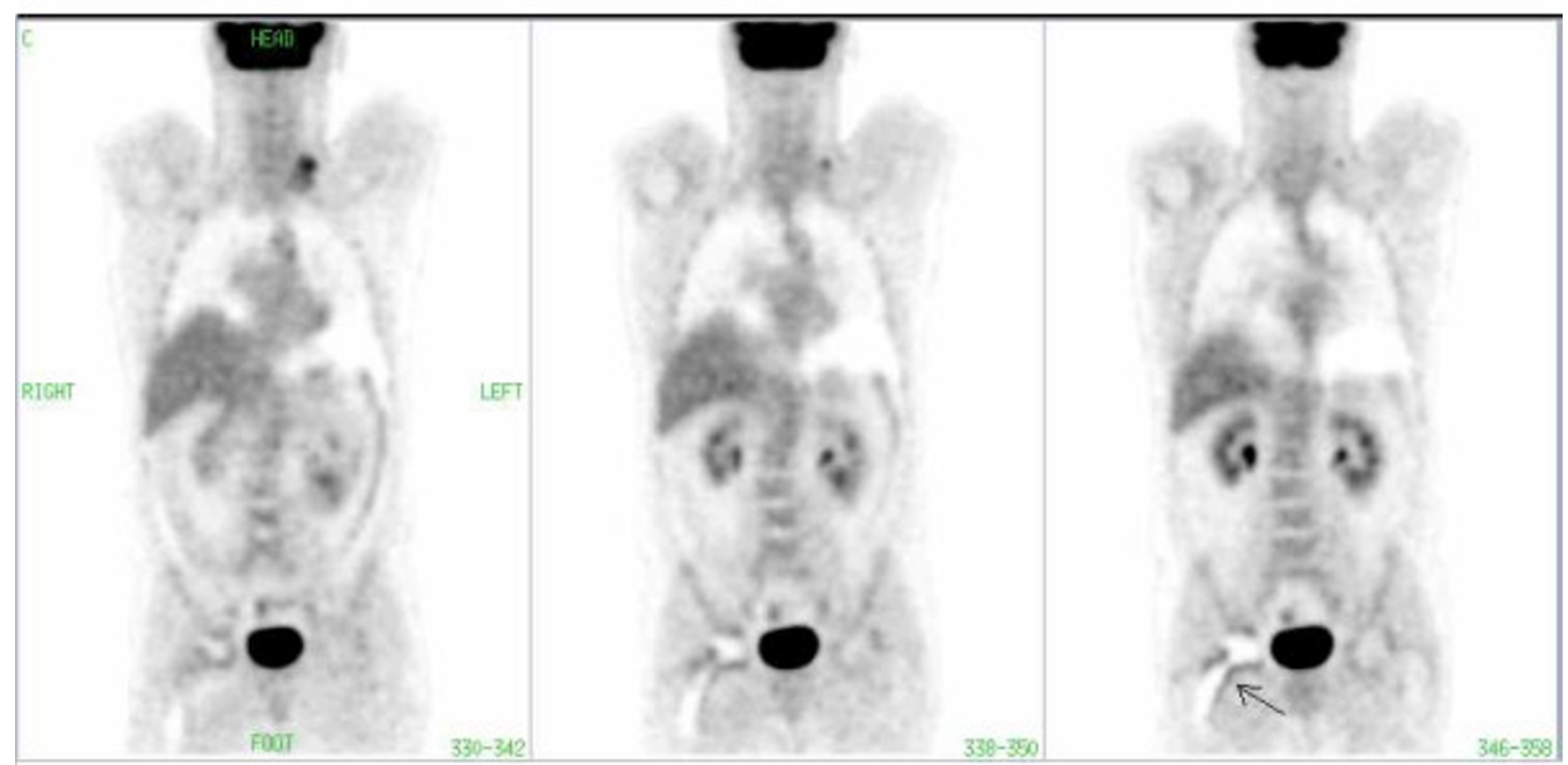

Figure 3

FDG-PET image of a patient with lymphoma involvement in the left cervical region. Note the periprosthetic artifact (arrow) in the region of the right hip in CT corrected images without segmentation.

measured transmission is used. Intermediate values result in a mix between the two. Default values were used $(0.50$ for lungs and 1.0 for soft tissues). Computation of $\mathrm{AC}$ with low dose CT data requires first the down-sampling of CT scan to PET matrix size and pixel size. Then CT truncation compensation is applied to compensate artifacts resulting in using a FOV of $600 \mathrm{~mm}$ [17]. Resolution matching is then performed by smoothing CT images. Gaussians filters are used with FWHMs that match PET resolution in axial and transaxial directions. CT images with pixels in Hounsfield Units (HU) are then converted to linear attenuation coefficients at $511 \mathrm{KeV}[18,19]$. Converted CT images are segmented to perform AC by dividing maps into regions representing tissue types, as described before. Images with $\mathrm{ACseg}^{+}$and ACseg- were displayed simultaneously in three different planes (coronal, sagittal, transaxial). Two nuclear medicine physicians reviewed the data sets with a specific multi-modality registration software (Philips Syntegra). PET and CT images can be visualized independently as well as registered on the same screen in coronal, sagittal and transverse planes. Layout can be customized and different presets are availa- ble to scale the CT images (lungs, soft tissues, bone, abdomen ...). Segmented transmissions scans obtained with Cs-137 and CT were also compared.

\section{Results}

The image quality in the area of metal implants was in all cases better with $\mathrm{ACseg}^{+}$than ACseg-, without artifacts seen in images with ACseg- (Figure 1, 2, 3, 4, 5). Further the emission images with $\mathrm{ACseg}^{+}$were qualitatively comparable to reconstructed data obtained with Cs-137 AC.

As shown in images 1-5 there are hypermetabolic areas seen in the ACseg- images in the area of metal implants which are not visible on $\mathrm{ACseg}^{+}$reconstructed images. Further we assessed the images without AC. Also in these images the artifacts due to metal implants were not visible, but the image quality was inferior to that of images with both methods of AC.

\section{Discussion}

For better anatomic localization of areas with increased tracer uptake in PET images, anato-metabolic imaging has 


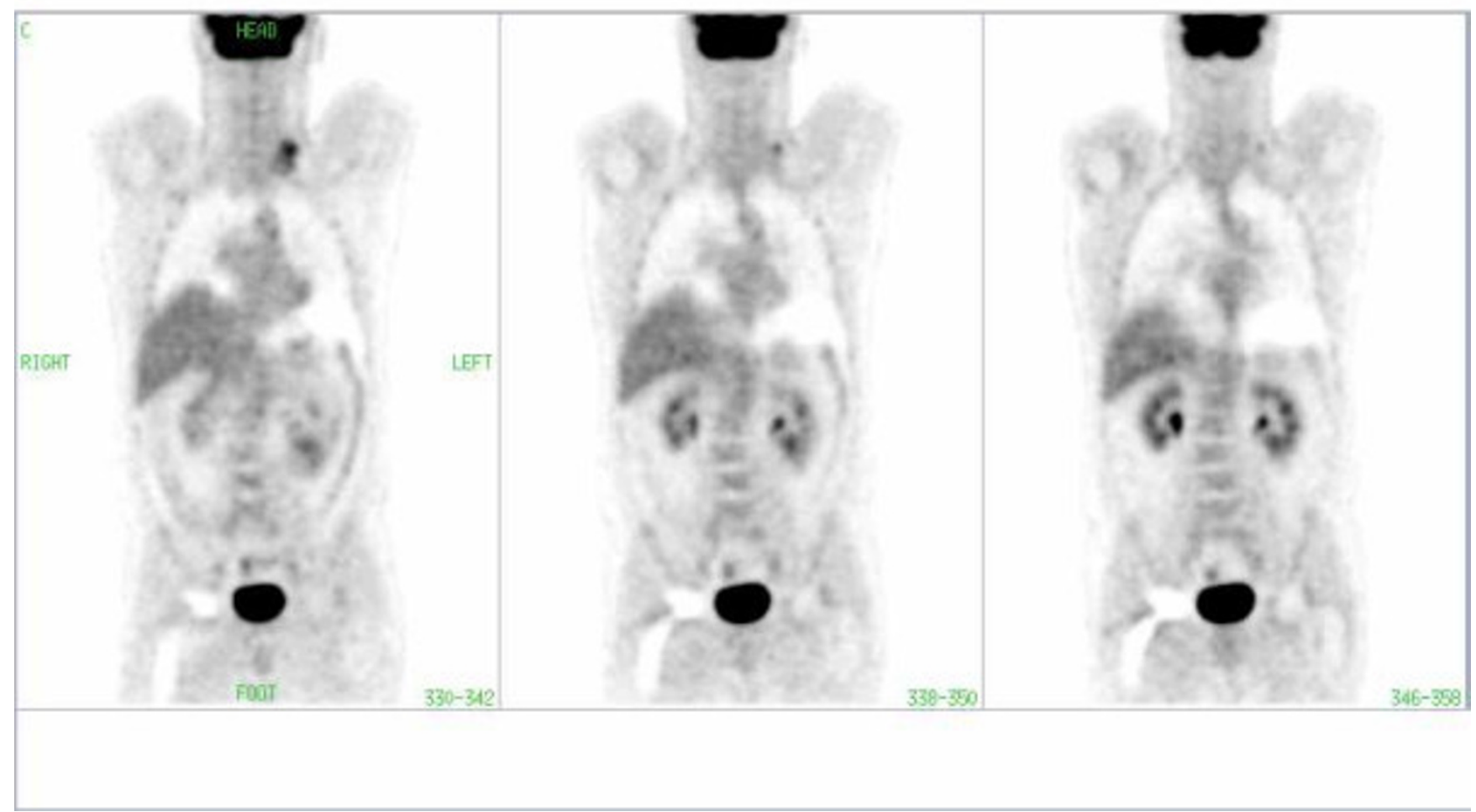

Figure 4

The same patient as in Fig. 3: There is no pathological uptake in the periprosthetic area of the right hip in the CT-corrected images with segmentation of transmission data.

been proposed during the same session, which has lead to manufacturing combined PET/CT devices [20]. This modality allows to obtain independently high quality PET and CT images as well as to merge both data sets to evaluate the morphological and functional information. For abdominopelvic evaluation, CT has been suggested as procedure of choice with PET imaging [21]. The CT transmission images are at the same time used for purpose of $\mathrm{AC}$ which is an indispensable part of image reconstruction and makes it also possible to get quantitative information in the areas of interest. There are three methods to generate attenuation maps from a CT image including segmentation, scaling and dual energy CT scans [22]. Oncologic patients often have artificial metal implants, such as metal braces in the spinal region, chemotherapy ports, dental fillings or implants, pacemaker, that can sometimes be mistaken for other pathologies $[23,24]$. PET transmission scans using point source of Cs-137 show little or no artifacts. With the significantly higher photon absorption at CT energy, however, these artifacts are present, which are not yet corrected in standard PET-CT protocols. Therefore the reconstruction of emission images without $\mathrm{AC}$ has been suggested $[22,25]$. As we could demonstrate in this study, the artifacts due to metal implants could be avoided using segmentation algorithm for AC with CT data. However, both methods for AC resulted in much better image quality compared to those without AC. In our center we did not make use of contrast medium for the CT part of the examination. But since the artifacts due to contrast medium in PET/CT images [26,27] are caused in the same way as CT hyperintense metal implants, we assume that these would also be avoided using AC with segmentation as described in our study.

\section{Conclusion}

Purpose of this study was to provide a qualitative comparison of attenuation corrected PET images of patients with metal implants produced by a PET-CT device with and without segmentation of CT. It is known that based on reconstruction methods there are differences in quantitative measurements because of the way transmission data are processed $[28,29]$. Further investigations should be performed to quantitatively estimate the effect of segmentation algorithms used for CT AC. 


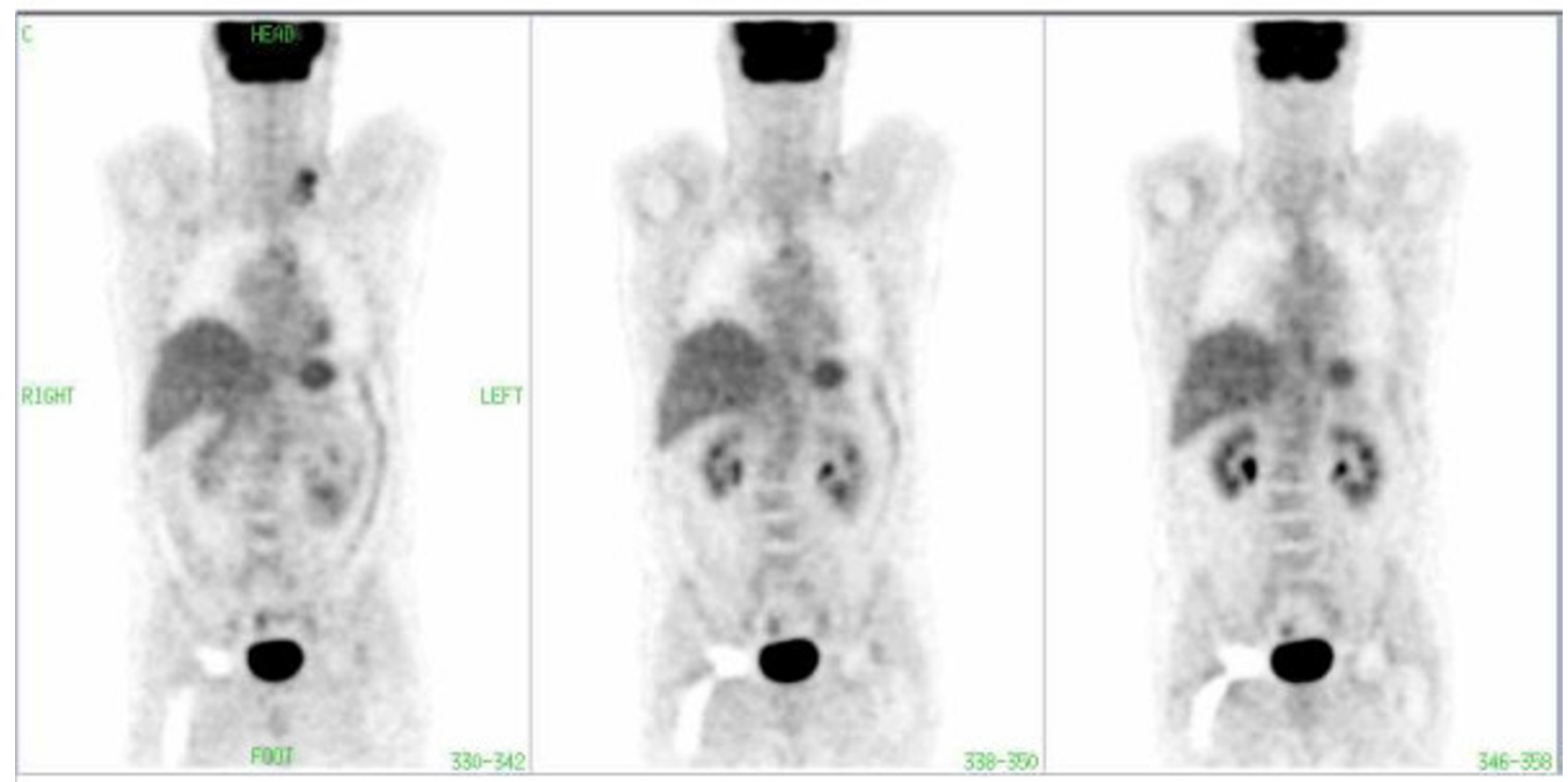

\section{Figure 5}

The same patient as in Fig. 3: There is no pathological uptake in the periprosthetic area of the right hip in the images corrected with $A C$ by $C s-137$.

In case of metal implants, we suggest that PET studies corrected by CT should preferably use the ACseg+ method to avoid the image artifacts.

\section{Abbreviations}

$\mathrm{AC}=$ Attenuation correction

Acseg- $^{-}$AC with non-segmented CT image

Acseg $^{+}=$AC with segmented CT image

CT = Computed Tomography

HU = Hounsfield Units

PET $=$ Positron Emission Tomography

\section{Competing interests}

The article-processing charge will be refunded by Philips. Dr Guerchaft is an employee of Philips Medical Systems. All other authors declare that they have no competing interests.

\section{Authors' contributions}

SM performed different reconstruction methods and drafted the manuscript. MG participated in drafting the manuscript and provided technical support. CB carried out the studies and participated in the design of the study. $\mathrm{PK}$ provided technical support. MD and $\mathrm{PB}$ participated in the design of the study. All authors read and approved the final manuscript.

\section{References}

I. Mayneord WV: The radiography of the human body with radioactive isotopes. BrJ Radiol 1952, 25:5 17-525.

2. Kuhl DE, Hale J, Eaton WL: Transmission scanning: a useful adjunct to conventional emission scanning for accurately keying isotope deposition to radiographic anatomy. Radiology 1966, 87:278.

3. Myers MJ, Lvender JP, deOliviera JB, Maseri A: A simplified method of quantitating organ uptake using a gamma camera. $\mathrm{Br} J$ Radiol 198I, 54:1062-1067.

4. Macey D, Marschall R: Absolute quantitation of radiotracer uptake in lungs using a gamma camera. J Nucl Med 1984, 23:73I-735.

5. Beyer T, Antoch G, Blodgett T, Freudenberg L, Akhurst T, Mueller S: Dual-modality PET/CT imaging: the effect of respiratory motion on combined image quality in clinical oncology. Eur J Nucl Med 2003, 30:588-596. 
6. Schaller S, Sembritzki O, Beyer T, Fuchs T, Kachelriess M, Flohr T: An algorithm for virtual extension of the CT field of measurement for application in combined PET/CT scanners. Radiology 2002, 225:497.

7. Dorio PJ, Lee FT, Henseler KP, Pilot M, Pozniak MA, Winter TC 3rd, Schock SA: Using a saline chaser to decrease contrast media in abdominal CT. AJR 2003, 180:929-934.

8. Goerres GW, Ziegler SI, Burger C, Berthold T, von Schultess GK, Buck A: Artifacts at PET and PET/CT caused by metallic hip prosthetic material. Radiol 2003, 226:577-584.

9. Goerres GW, von Schultess GK, Hany TF, Kamel EM, Burger C, Buck A, Schultess GKv, Goerres GW: Impact of metallic dental implants on CT-based attenuation correction in a combined PET/CT scanner. Eur Radiol 2002, I3:724-728.

10. Nehmeh SA, Erdi YE, Kalaigian H, Kolbert KS, Pan T, Yeung H, Squire O, Sinha A, Larson SM, Humm JL: Correction for Oral Cntrast Artifacts in CT Attenuation-Corrected PET Images Obtained by Combined PET/CT. I Nucl Med 2003, 44:1940-1944.

II. Townsend DW, Beyer T, Blodgett TM: PET/CT scanners: a hardware approach to image fusion. Semin Nucl Med 2003, 33:193-204.

12. Daube-Witherspoon ME, Matej S, Karp JS, Lewitt RM: Application of the row action maximum likelihood algorithm with spherical basis functions to clinical PET imaging. Trans Nucl Sci 200I, 48:24-30.

13. Takashi Obi, Matej S, Lewitt RM, Gabor Herman T: 2.5 D Simultaneous Multislice Reconstruction by Series Expansion Methods from Fourier-Rebinned PET data. IEEE Trans Med Img 2000, 19:474-484.

14. Browne J, De Pierro AR: A row-action alternative to the EM algorithm for maximizing likelihood in emission tomography. IEEE Trans Med Img 1996, 15:687-699.

15. Nock R, Nielsen F: Statistical region merging. IEEE Trans Pattern Anal Mach Intell 2004, 26:1452-1458.

16. Ray N, Acton ST, Altes T, de Lange EE, Brookman JR: Merging parametric active contours within homogenous image regions for MRI-based lung segmentation. IEEE Trans Med Imaging 2003, 22:189-199.

17. Bai C, Brown KM, Shao L, Da Silva AJ, Zhao Z: A new technique for CT truncation compensation in combined CT/PET imaging. (Abstract) J Nucl Med 2003, 44:270.

18. Bai C, Shao L, Da Silva AJ, Zhao Z: A generalized model for the conversion from $C T$ numbers to linear attenuation coefficients. IEEE Trans Nucl Sci 2003, 50:15 I0-15I5.

19. Bai C, Shao L, Da Silva AJ, Zhao Z, Hines H: Low-dose CT scans for CT-based attenuation correction in PET/CT Imaging. (Abstract) J Nucl Med 2002, 43: 104.

20. Beyer T, Kinahan PE, Townsend DW, Sashin D: The use of X-ray CT for attenuation correction of PET data. In Proceedings of the 1994 IEEE Nuclear Science Symposium and Medical Imaging Conference Volume 4. Edited by: Trendler RC. Norfolk, VA, USA; 1994:1573-1577.

21. Wahl RL: Why nearly all PET of abdominal and pelvic cancers will be performed as PET/CT. J Nucl Med 2004, 45:82-95.

22. Kinahan PE, Hasegawa BH, Beyer T: X-Ray-Based Attenuation Correction for Positron Emission Tomography/Computed Tomography Scanners. Sem Nucl Med 2003, 3:166-179.

23. Shreve PD, Anzai Y, Wahl RL: Pitfalls in oncologic diagnosis with FDG PET imaging: physiologic and benign variants. RadioGraphics 1999, 19:61-77.

24. Beyer Th, Antoch G, Müller S, Egelhof T, Freudenberg LS, Debatin J, Bockisch A: Acquisition protocol consideration for combined PET/CT imaging. J Nucl Med 2003, 45:25-35.

25. Kamel EM, Burger C, Buck A, von Schulthess GK, Goerres GW: Impact of metallic dental implants on CT-based attenuation correction in a combined PET/CT scanner. Eur Radiol 2003, 13:724-8.

26. Cohade C, Osman M, Nakamoto Y, Marschall LT, Leal JP, Wahl RL: Initial experience with oral contrast in PET/CT: phantom and clinical studies. J Nucl Med 2003, 44:4I2-416.

27. Antoch G, Freudenberg LS, Beyert T, Bockisch A, Debatin JF: To enhance or not to enhance? I8F-FDG and CT contrast agents in dual-modality I8F-FDG PET/CT. J Nucl Med 2004, 45:56-65.
28. Schrhröder H, Erdi YE, Chao K, Gonen M, Larson SM, Yeung HW: Clinical implication of different image reconstruction parameters for interpretation of whole-body PET studies in cancer patients. I Nucl Med 2004, 45:559-566.

29. Visvikis D, Cheze-LeRest C, Costa DC, Bomanji J, Gacinovic S, Ell PJ: Influence of OSEM and segmented attenuation correction in the calculation of standardised uptake values for [ $\left.{ }^{18} \mathrm{~F}\right] \mathrm{FDG}$ PET. Eur J Nucl Med 200I, 28:I326-I 335.

\section{Pre-publication history}

The pre-publication history for this paper can be accessed here:

\section{http://www.biomedcentral.com/1471-2385/5/3/prepub}

Publish with Biomed Central and every scientist can read your work free of charge

"BioMed Central will be the most significant development for disseminating the results of biomedical research in our lifetime. "

Sir Paul Nurse, Cancer Research UK

Your research papers will be:

- available free of charge to the entire biomedical community

- peer reviewed and published immediately upon acceptance

- cited in PubMed and archived on PubMed Central

- yours - you keep the copyright

Submit your manuscript here:

http://www.biomedcentral.com/info/publishing_adv.asp
BioMedcentral 